\title{
Simulación Discreta Aplicada Al Proceso Productivo De Vestidos De Baño En Colombia
}

\section{Discrete Event Simulation Applied to Productive Process of Swimsuits in Colombia}

Carlos M. Echavarría ${ }^{1}$, Lis A. Piedrahíta², Daniela Ramírez ${ }^{3}$ Yony F. Ceballos ${ }^{4}$

\begin{abstract}
RESUMEN
La simulación es una herramienta que permite modelar un gran número de sistemas de la vida cotidiana y proponer acciones de mejora a un bajo costo. Gracias a esto se ha aplicado en varios sectores disminuyendo costos y tiempos. En la producción de trajes de baño en una empresa reconocida del sector manufacturero se presentan cuellos de botella y por lo tanto, se construyó un modelo de simulación que permitiera analizar, proponer y evaluar alternativas para mejorar el proceso de confección, haciendo énfasis en el subproceso de decoración que según información histórica de la compañía, era donde se presentaban mayores demoras retrasando así los tiempos de entrega de producto terminado. Se inició con una descripción del proceso productivo, una toma de datos y visitas guiadas a la planta de trabajo. Posteriormente se elaboró un modelo de simulación del proceso de producción con el ánimo de evidenciar en el modelo propuesto la situación actual y de ahí, proponer diferentes escenarios y recomendaciones basados en las modificaciones económica y físicamente factibles para la empresa. Se concluye que se deben contratar dos nuevos empleados para solucionar el cuello de botella en el proceso de decorado y además se hacen un conjunto de sugerencias que pueden llevarse a cabo cuando la empresa decida ampliarse, dejando en la misma un modelo dinámico que puede cambiarse de forma simple en caso de ser requerido.
\end{abstract}

PALABRAS CLAVES: Simulación, línea de producción, cuello de botella, confección de vestidos de baño, sistema de colas.

\footnotetext{
${ }^{1}$ Estudiante ingeniería industrial, Universidad de Antioquia, Medellín - Antioquia - Colombia, carlosm.echavarria@udea.edu.co

2 Estudiante ingeniería industrial, Universidad de Antioquia, Medellín - Antioquia - Colombia, lis.piedrahita@udea.edu.co

${ }^{3}$ Estudiante ingeniería industrial, Universidad de Antioquia, Medellín - Antioquia - Colombia, Daniela.ramirez12@udea.edu.co

4 *Doctor en Ingeniería e Ingeniero de sistemas e informática, profesor del departamento de ingeniería industrial en la Universidad de Antioquia, Calle 67 No. 53 - 108 oficina 21-407 teléfono 2195575, fax 2638282, Medellín - Antioquia - Colombia yony.ceballos@udea.edu.co
} 


\begin{abstract}
Simulation is a tool that allows modelling a large number of systems of daily life and propose improvement actions at a low cost. Thanks to this has been applied in various sectors reducing costs and times. In the production of swimwear in a renowned company of manufacturing bottlenecks occur and therefore, a simulation model that would analyse, propose and evaluate alternatives to improve the process was built, making emphasis on decoration thread as historical information of the company, was where further delays presented thus delaying delivery times of finished product. It began with a description of the production process, a data collection and guided visits to the company. A simulation model of the production process with the aim of demonstrating the proposed model and the current situation there is then prepared to propose different scenarios and recommendations based on economic and physically changes feasible for the company. It is concluded to be hired two new employees to solve the bottleneck in the process arranged and a set of suggestions that can be done are done when the company decides to expand, leaving a dynamic model that can be changed in a simple way if it is required.
\end{abstract}

KEYWORDS: Simulation, production line, bottleneck, swimsuits confection, Queuing system.

\section{INTRODUCCIÓN}

En la actualidad la simulación se ha convertido en parte importante para la toma de decisiones de una empresa sin importar sus dimensiones. Sin embargo, son muy escasos los artículos que presentan procesos de manufactura de trajes de baño con fases artesanales en su elaboración. Estas fases están asociadas a la decoración de las prendas, y son diseños únicos de los operarios encargados de realizarlos. El resto del proceso de confección es similar a otros procesos de elaboración de vestidos de baño.

En cuanto al estado del arte, se encuentra que en países como Brasil, Argentina, Ecuador y Colombia se realizan análisis similares, relacionados con producción, que presentan la implementación de modelos de simulación discreta en una fábrica de pulpa de frutas y otro estudio en la elaboración de té seco, mejorando ambos procesos (Quiroga et al. 2009; Michalus et al. 2007). Otro estudio asociado a la producción de bioetanol se analiza mediante el uso de simulación, empleando residuos agroindustriales para la explotación de aditivos para combustible, disminuyendo el desperdicio de materias primas. Se concluye que la simulación es una herramienta útil en la simulación de procesos asociados a productos perecederos (Morales et al. 2010). Por último se encuentra el trabajo de la Universidad del Valle "Modelo de simulación del abastecimiento de biomasa de caña de azúcar como sistema de soporte a las decisiones de cosecha" en el cual se realiza un estudio de la manipulación de materias orgánicas de desecho y se sugieren varios escenarios de solución, en este caso se emplea el software C++ (Bocanegra 2013).

En cuanto a manufactura de prendas de vestir, se realiza en la empresa Creatum Accesorios S.A. un análisis que involucra la optimización del proceso de estampado y bordado de marquillas y se dan recomendaciones orientadas a mejorar el proceso en fases posteriores asociadas al crecimiento de la misma (Zapata Gaviria \& Peña 2006). Posteriormente se hace un estudio de simulación para mejorar la gestión de pedidos en la empresa Imagen Textil, logrando optimizar el proceso de entrega de prendas (Arequipa 2013).

En vista de lo anterior, se identifica que el empleo de Simul8® como software de simulación discreta es versátil y que puede aplicarse en diversas industrias, asociadas a productos perecederos y maquila de prendas.

En el caso de estudio, la producción de vestidos de baño es llevada a cabo durante los meses Enero, Abril, Junio, Julio y Diciembre, en los cuales se reporta un tope máximo de producción equivalente a 30.000 prendas. Para obtener dichas prendas es necesario pasar por una serie de etapas antes de entregar el producto final; tales como: Trazo, Corte, Confección, Diseño, Pulido y empaque; sin embargo es necesario aclarar que dentro de la organización no se lleva a cabo cada etapa, debido a que se tienen convenios con terceros en Riosucio (Caldas), los cuales se encargan de realizar la decoración y empaque de las prendas; es allí precisamente donde se presenta el problema para la empresa, anteriormente se contaba con un solo operario el cual se encargaba de la decoración de los vestidos 
de baño, se determinó a raíz de un estudio realizado que se debía incrementar este número con lo cual se aumentó a cinco operarios. Teniendo en cuenta que se cuentan con cinco referencias de vestidos de baño las cuales deben ser entregadas a sus clientes por pedidos. Se debe tener en cuenta que la mano de obra es bastante económica en el municipio donde se lleva a cabo el proceso de decoración.

Por lo tanto, el objetivo principal es realizar un modelo el cual permita evaluar alternativas de mejora en el proceso de decorado de los vestidos de baño; mediante la evaluación de los diferentes métodos de trabajo al interior de la compañía y minimizar el tiempo de decorado en los lotes e identificar los cuellos de botella presentes en el proceso. Se pretende realizar un modelo que sea dinámico y que permita la inclusión de nuevas variables, eventos, esperas al sistema y otras eventualidades.

\section{MATERIALES Y MÉTODOS}

\subsection{Materiales}

Los materiales utilizados para hacer el modelo y la investigación fueron: Software Simul8®, bases de datos según algunos históricos que tenía la compañía, toma de datos por parte de los empleados de la empresa, además se usó el software Statgraphics $₫$ para realizar las pruebas de bondad y ajuste para las distribuciones de las variables que se mencionarán a lo largo del artículo.

\subsection{Metodología}

Para elaborar la simulación se siguieron los pasos propuestos por diferentes referentes teóricos como textos de estudio y otros artículos de aplicación (Avila \& Vásquez 2008; Banks 1998; Michalus et al. 2007).

2.2.1 Definición del problema: el estudio se enfocó en el proceso de decorado de la prenda confeccionada, ello debido a las constantes quejas hechas por sus clientes, las cuales son causadas en su mayoría por el tiempo de entrega de los vestidos de baño; puesto que se tiene un cuello de botella en este proceso, lo cual impide el normal funcionamiento de la cadena de producción. Esta situación afecta significativamente los ingresos de la empresa, dado que al represarse la producción, los pedidos son enviados a destiempo y se corre el riesgo de que sean devueltos, el pedido sea cancelado y que el cliente cambie de proveedor.

Además de lo dicho anteriormente, es importante anotar que el proceso tiene una capacidad infinita, ya que la empresa no presenta restricciones en cuanto a la cantidad de lotes almacenados, pues se produce sobre pedido, y de esta manera la producción fluye constantemente; al trabajar sobre pedido la empresa no tiene un control total sobre los lotes que ingresan, es por esto que se deben utilizar herramientas probabilísticas que ayuden al análisis de dicho evento, debido a la aleatoriedad del ingreso de los lotes al proceso de decorado.

Luego de identificar la problemática se evidencia que es necesario implementar un modelo de simulación apoyado en el método de teoría de colas; para su aplicación se debe analizar la entrada diaria de lotes y el tiempo que requiere un operario en realizar la labor de decorado, esto para conocer la tasa de llegada de los lotes y de servicio de la empresa, además, es necesario conocer los costos que incurren en este proceso, de modo que se pueda desarrollar el proceso eficientemente y al mismo tiempo se disminuyan los costos totales atribuidos al mismo. Además, se estudiará de acuerdo a los resultados obtenidos la posibilidad de proceder a solucionar este problema con contrataciones en temporadas altas, capacitación del personal, inversión en tecnología y/o mejoras de métodos y tiempos de trabajo.

2.2.2 Descripción del proceso: El proceso de fabricación y decorado de vestidos de baño inicia con la entrada de las materias primas, las cuales son lotes de tela y rollos de papel; las telas son extendidas sobre la mesa de corte mientras otro operario se encarga de trazar las prendas (moldes) en el papel, el cual es llevado a la sala donde se encuentra las telas. Después de esto, se procede a realizar el proceso de corte del vestido de baño ya trazado, la cual se lleva a cabo mediante una máquina, estos cortes son trasladados a la zona de pulido donde cinco operarios se encargan de retocar las prendas.

Al terminar en la zona de pulido se procede a trasladar los lotes de las referencias a la zona de armado donde se tiene una llegada de hilos para cada máquina, luego de realizar esta actividad se procede a realizar el traslado del lote producido al camión que transporta dicho lote a Riosucio Caldas donde se encargan de realizar el decorado de las prendas, se cuenta con una llegada de material para decorado donde dicho material es separado de acuerdo a cada referencia, material que es trasladado al área de decorado y es entregado a cada operario, además de esto se les entregan los lotes de cada referencia y se procede a realizar la actividad de decorado, después es recolectada cada referencia y se lleva al área de empaque donde un operario se encarga de realizar esta labor. Al culminar todas las actividades los lotes son entregados al cliente. 
Para contextualizar un poco más acerca de cómo se realizó la toma de datos, construcción del modelo y variables se explicarán a continuación la terminología usada y las variables escogidas, además de una tabla con el resumen de las mismas y su forma de medición.

2.2.3 Definición de las variables: Con el fin de identificar las variables y elementos que actúan en el sistema o proceso, es necesario clasificar dichas variables en tres tipos: Exógenas, Endógenas y de Estado, además de dar la definición de conceptos afines al proceso a estudiar.

Una variable es un elemento que varía o cambia con el tiempo, es decir, es algo que suele ser inestable, mudable e inconstante. Las variables dentro de la simulación suelen clasificarse en:

Variables Exógenas: Representan acciones influenciadas por el medio ambiente 0 agentes externos al proceso, por lo tanto no son controlables; sin embargo, cuando las variables exógenas son controlables por el sistema se denominan parámetros y asumen valores fijos; las variables exógenas definidas para este proceso son:

Tiempo de trazo: Es el tiempo empleado por lo operarios para calcar en papel los moldes de cada referencia de los vestidos de baño.

Tiempo de corte: Es el tiempo que se requiere para cortar la tela con el molde correspondiente.

Tiempo de pulido: Es el tiempo empleado para pulir el corte de las referencias.

Tiempo de armado: Esta variable representa el tiempo requerido para unir los cortes anteriormente mencionados con ayuda de un hilo. Tiempo de decorado: Es el tiempo usado para personalizar cada referencia.

Tiempo de empacado: Es el tiempo requerido para empacar cada referencia de acuerdo a la demanda establecida por cada referencia.

Número de unidades a producir: Hace referencia a la demanda requerida por los proveedores de cada referencia, teniendo presente que un lote está completo siempre y cuando el número pedido de cada modelo esté terminado.

Variables Endógenas: Son aquellas que representan una actividad que e da internamente en el sistema; las variables exógenas definidas para este proceso son:

Número de prendas en el sistema: Se refiere al número de vestidos de baño que quedan en proceso en el omento que se finaliza el tiempo o plazo de producción que en simul8 sería las prendas que quedan sin terminar al momento que se completa el tiempo de simulación.

Número de prendas producidas por día: Es el número de vestidos de baño terminados por día.
Variables de Estado: Las variables de estado se derivan de las endógenas, las cuales muestran o reflejan características relevantes del sistema y de los elementos que lo componen en el instante $t$ que se observen. Las variables de estado definidas para este proceso son:

Tiempo de espera de cada referencia en el sistema: Es el tiempo que tardan en cola cada referencia a lo largo de todo el proceso de fabricación.

Número de servidores óptimo para el proceso de decorado: Es la variable que se quiere estimar para finalmente dar recomendaciones a la compañía.

2.2.4 Conceptualización del modelo: El proceso de fabricación y decoración de vestidos de baño de la empresa de confecciones, se realiza mediante los siguientes pasos:

La tela entra a la fábrica y se clasifica dependiendo si es para el forro y para el encaje, después de realizar esto se determinan las telas que el cliente ha escogido con antelación. En la zona de cortado el encargado extiende tela por tela para evitar que se arruguen y se dañen al realizar esta actividad, paralelo a esto se tiene otra persona que se encarga de trazar en el papel las referencias que el cliente solicitó, este es llevado a la zona de corte donde ya se tiene las telas extendidas en la mesa; hay que tener en cuenta que siempre se realiza un solo corte por cliente independiente de la cantidad solicitada ya que la maquina tiene capacidad infinita, luego de tener los corte realizados se procede a llevar las piezas clasificadas por referencia al área de pulido donde se realiza un análisis rápido a los producto, se perfeccionan y perfilan las referencias, las cuales son pasadas al área de armando donde se cuentan con cinco operarios, quienes se encargan de organizar y confeccionar los vestidos de baño.

Luego de tener todo el lote completo este es enviado a municipio de Riosucio departamento de caldas donde se encuentran las decoradoras. En esta parte del proceso las decoradoras tienen los diseños que el cliente escogió y se procede a realizar dicha actividad, decorando tanto el brasier como la tanga según las especificaciones requeridas. Al terminar todo en área de decorado, se envía al almacén de empaques donde se pone la marquilla, talla y se empaca el vestido en bolsas transparentes. Se debe de tener todo el lote completo puesto que se debe entregar el pedido completo al cliente.

Recopilación y modelado de datos: La mayoría de datos de entrada fueron tomados con base a los pedidos que realiza un cliente (el cual es de 120 unidades) los datos de tiempo de armado de 
vestido y tiempo de pulido fueron tomados por nosotros en la compañía y ajustados de distribuciones, otros datos son valores fijos dados por el coordinador del proceso de elaboración de los vestidos de la compañía y para el proceso de decoración (la cual es realizada en el municipio de Riosucio) Un empleado de la compañía fue el encargado de realizar la toma de los datos; todos los datos fueron tomados en minutos. En la siguiente tabla se mostrará más claramente cómo se realizó la toma de los datos:

Tabla 1: Datos requeridos para el modelo de simulación

\begin{tabular}{|c|c|c|}
\hline $\begin{array}{l}\text { Nombre } \\
\text { del dato }\end{array}$ & $\begin{array}{l}\text { Forma en que se } \\
\text { realizó la } \\
\text { medición }\end{array}$ & $\begin{array}{l}\text { Distribución } \\
\text { ajustada o } \\
\text { valor fijo(en } \\
\text { minutos) } \\
\end{array}$ \\
\hline $\begin{array}{l}\text { Tiempo de } \\
\text { trazo }\end{array}$ & $\begin{array}{l}\text { Número fijo ya } \\
\text { que la máquina } \\
\text { demora un tiempo } \\
\text { estándar }\end{array}$ & $\begin{array}{l}\text { Valor fijo de } 60 \\
\text { minutos }\end{array}$ \\
\hline $\begin{array}{l}\text { tiempo de } \\
\text { corte }\end{array}$ & $\begin{array}{l}\text { Número fijo ya } \\
\text { que la máquina } \\
\text { demora un tiempo } \\
\text { estándar }\end{array}$ & $\begin{array}{l}\text { Valor fijo de } 120 \\
\text { minutos }\end{array}$ \\
\hline $\begin{array}{l}\text { Tiempo de } \\
\text { pulido } \\
\text { referencia } 1\end{array}$ & $\begin{array}{l}\text { Toma de datos y } \\
\text { ajuste de } \\
\text { distribuciones. }\end{array}$ & $\begin{array}{l}\text { Triangular } \\
\text { Límite superior: } \\
5,1 \text { límite } \\
\text { inferior: } 4,1 \\
\text { Media: } 4,15\end{array}$ \\
\hline $\begin{array}{l}\text { Tiempo de } \\
\text { pulido } \\
\text { referencia } 2\end{array}$ & $\begin{array}{l}\text { Toma de datos y } \\
\text { ajuste de } \\
\text { distribuciones. }\end{array}$ & $\begin{array}{l}\text { Weibull } \\
\alpha: 20,74 \\
\beta: 4,52\end{array}$ \\
\hline $\begin{array}{l}\text { Tiempo de } \\
\text { pulido } \\
\text { referencia } 3\end{array}$ & $\begin{array}{l}\text { Toma de datos y } \\
\text { ajuste de } \\
\text { distribuciones. }\end{array}$ & $\begin{array}{l}\text { Gamma } \\
\alpha: 575,88 \\
\beta: 132,85\end{array}$ \\
\hline $\begin{array}{l}\text { Tiempo de } \\
\text { pulido } \\
\text { referencia } 4\end{array}$ & $\begin{array}{l}\text { Toma de datos y } \\
\text { ajuste de } \\
\text { distribuciones. }\end{array}$ & $\begin{array}{l}\text { Weibull } \\
\alpha: 16,38 \\
\beta: 4,52\end{array}$ \\
\hline $\begin{array}{l}\text { Tiempo de } \\
\text { pulido } \\
\text { referencia } 5\end{array}$ & $\begin{array}{l}\text { Toma de datos y } \\
\text { ajuste de } \\
\text { distribuciones. }\end{array}$ & $\begin{array}{l}\text { Normal } \\
\mu: 4,35 \\
\sigma: 0,13\end{array}$ \\
\hline $\begin{array}{l}\text { Tiempo de } \\
\text { armado } \\
\text { referencia } 1\end{array}$ & $\begin{array}{l}\text { Toma de datos y } \\
\text { ajuste de } \\
\text { distribuciones. }\end{array}$ & $\begin{array}{l}\text { Uniforme } \\
\text { Límite superior: } \\
27,43 \\
\text { Límite inferior: } \\
20\end{array}$ \\
\hline $\begin{array}{l}\text { Tiempo de } \\
\text { armado } \\
\text { referencia } 2\end{array}$ & $\begin{array}{l}\text { Toma de datos y } \\
\text { ajuste de } \\
\text { distribuciones. }\end{array}$ & $\begin{array}{l}\text { Triangular } \\
\text { Límite } \\
\text { superior:31,36 } \\
\text { Límite inferior: } \\
20,01 \\
\text { Media: } 20,01\end{array}$ \\
\hline $\begin{array}{l}\text { Tiempo de } \\
\text { armado } \\
\text { referencia } 3\end{array}$ & $\begin{array}{l}\text { Toma de datos y } \\
\text { ajuste de } \\
\text { distribuciones. }\end{array}$ & $\begin{array}{l}\text { Lognormal } \\
\mu: 26,71 \\
\sigma: 5,9\end{array}$ \\
\hline
\end{tabular}

\begin{tabular}{|c|c|c|}
\hline $\begin{array}{l}\text { Tiempo de } \\
\text { armado } \\
\text { referencia } 4\end{array}$ & $\begin{array}{l}\text { Toma de datos y } \\
\text { ajuste de } \\
\text { distribuciones. }\end{array}$ & $\begin{array}{l}\text { Weibull } \\
\alpha: 25,33 \\
\beta: 10,69\end{array}$ \\
\hline $\begin{array}{l}\text { Tiempo de } \\
\text { armado } \\
\text { referencia } 5\end{array}$ & $\begin{array}{l}\text { Toma de datos y } \\
\text { ajuste de } \\
\text { distribuciones. }\end{array}$ & $\begin{array}{l}\text { Lognormal } \\
\mu: 27.5325 \\
\sigma: 3,19184\end{array}$ \\
\hline $\begin{array}{l}\text { Tiempo de } \\
\text { decorado } \\
\text { referencia } 1\end{array}$ & $\begin{array}{l}\text { Toma de datos } \\
\text { por parte de un } \\
\text { empleado y ajuste } \\
\text { de distribuciones. }\end{array}$ & $\begin{array}{l}\text { Uniforme } \\
\text { Límite superior: } \\
310,2 \\
\text { Límite inferior: } \\
300,5\end{array}$ \\
\hline $\begin{array}{l}\text { Tiempo de } \\
\text { decorado } \\
\text { referencia } 2\end{array}$ & $\begin{array}{l}\text { Toma de datos } \\
\text { por parte de un } \\
\text { empleado y ajuste } \\
\text { de distribuciones. }\end{array}$ & $\begin{array}{l}\text { Uniforme } \\
\text { Límite superior: } \\
311,5 \\
\text { Límite inferior: } \\
300,2\end{array}$ \\
\hline $\begin{array}{l}\text { Tiempo de } \\
\text { decorado } \\
\text { referencia } 3\end{array}$ & $\begin{array}{l}\text { Toma de datos } \\
\text { por parte de un } \\
\text { empleado y ajuste } \\
\text { de distribuciones. }\end{array}$ & $\begin{array}{l}\text { Uniforme } \\
\text { Límite superior: } \\
309,4 \text { límite } \\
\text { inferior: } 300,1\end{array}$ \\
\hline $\begin{array}{l}\text { Tiempo de } \\
\text { decorado } \\
\text { referencia } 4\end{array}$ & $\begin{array}{l}\text { Toma de datos } \\
\text { por parte de un } \\
\text { empleado y ajuste } \\
\text { de distribuciones. }\end{array}$ & $\begin{array}{l}\text { Uniforme } \\
\text { Límite superior: } \\
312,4 \\
\text { Límite inferior: } \\
301,5\end{array}$ \\
\hline $\begin{array}{l}\text { Tiempo de } \\
\text { decorado } \\
\text { referencia } 5\end{array}$ & $\begin{array}{l}\text { Toma de datos } \\
\text { por parte de un } \\
\text { empleado y } \\
\text { ajuste. }\end{array}$ & $\begin{array}{l}\text { Uniforme } \\
\text { Límite superior: } \\
310,2 \\
\text { Límite inferior: } \\
305,5\end{array}$ \\
\hline $\begin{array}{l}\text { Tiempo de } \\
\text { empacado }\end{array}$ & $\begin{array}{l}\text { Toma de datos } \\
\text { por parte de un } \\
\text { empleado y ajuste } \\
\text { de distribuciones }\end{array}$ & $\begin{array}{l}\text { Uniforme } \\
\text { Límite superior: } \\
3 \\
\text { Límite inferior: } 2\end{array}$ \\
\hline $\begin{array}{l}\text { Número de } \\
\text { unidades a } \\
\text { producir }\end{array}$ & $\begin{array}{l}\text { Número fijo } \\
\text { brindado por el } \\
\text { coordinador del } \\
\text { proceso }\end{array}$ & $\begin{array}{l}\text { Valor fijo de } 120 \\
\text { unidades }\end{array}$ \\
\hline
\end{tabular}

Fuente: Elaboración propia

No se realizó muestro aleatorio debido a que en algunas partes del proceso las unidades solicitadas por el cliente deben estar completas para entrar a otra actividad lo cual hace complejo el desarrollo del muestreo en este proceso, por cual se toma como muestra las 120 unidades solicitadas por el cliente las cuales se encuentran dividida en 5 referencias, cada una con 24 unidades.

\subsection{Elaboración de un modelo formal}

Para obtener un modelo formal de simulación mediante el software Simul $8 \AA$, se hizo uso de la información descrita en la conceptualización de dicho modelo, además de la recopilación y análisis de los datos; lo cual desencadena como resultado el modelo observado en la figura 1. 


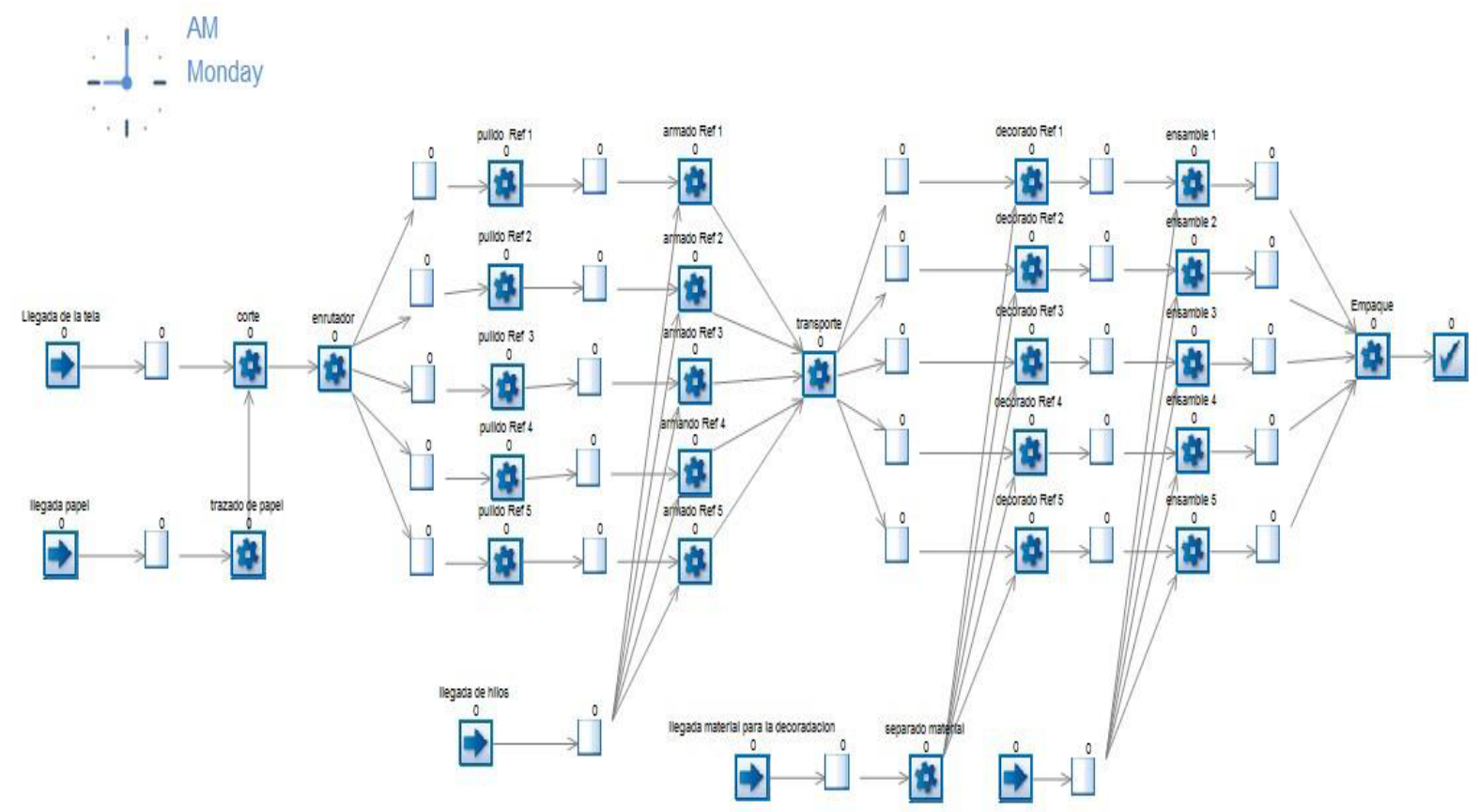

Figura 1: Modelo general implementado en Simul8®. Fuente: Elaboración propia

Se observa entonces en la figura 1 el proceso productivo como tal de los vestidos de baño teniendo sus 4 entradas que son: tela, papel, hilos, material para decoración y las bolsas para empacar los vestidos; también se tienen las actividades de: corte, trazado de papel, pulido, armado, transporte, decorado, ensamble, empaque, además de las respectivas esperas y en último lugar la salida completa del lote de 124 unidades.

Para verificar si el modelo elaborado está acorde con la situación real, se realiza un proceso de verificación, el cual está orientado a corregir errores en cuento a la lógica operacional del programa Para ello se empleó la prueba de degeneración (Banks 1998; Gilbert \& Troitzsch 2005).

Por lo tanto, se procedió a variar las tasas de llegada de la tela, el papel de trazado, el hilo, el material de decoración y las bolsas para el ensamble, con lo cual se encontró que a medida que se aumentan dichas entradas se generan colas en los procesos de armado, decorado y ensamble, por lo tanto no es posible cubrir la demanda solicitada, aspecto que también se vive en el sistema real, ya que cuando están en temporada alta la demanda sube, pero la compañía se ve en apuros para cumplir a tiempo y satisfactoriamente con dicho pedido.

En cuanto a la validación, se realiza un procedimiento de validez de los eventos, el cual, para las 5 referencias de vestidos se obtuvo que en el sistema simulado se produjeron 119 unidades en total con una $\mu=23.8$ y $\sigma=0.44$ y para el sistema real se obtuvo como resultado 114 unidades en total de las cinco referencias con $\mu=$ 23.2 y $\sigma=0.440 .84$ se observa entonces que la diferencia es aceptable, a pesar de que en la simulación los lotes en su mayoría salen completos, se puede determinar que el sistema simulado tiene una alta confiabilidad pues en él se plasma de forma concisa las evidencias del sistema real; lo cual se comprobará con la validez externa.

Para la validez externa, se consultaron a los expertos del proceso; las personas encargadas de coordinar la producción, quiénes analizaron el funcionamiento de la simulación y concluyeron que este se comportaba de manera similar al real, además agregaron que sería de gran utilidad realizar la simulación para más lotes pues se podría visualizar mejor el trabajo realizado; sin embargo, fueron conscientes de que la toma de los datos para ejecutar este cambio es dispendiosa. Se resaltó que a partir del modelo se podrían generar recomendaciones importantes para el mejoramiento del proceso pues idealiza el sistema real.

En cuanto a la sensibilidad del modelo elaborado, se realizaron varias iteraciones cambiando las entradas del proceso las cuales son: entrada de tela, entrada de hilos, entrada de papel, entrada de material para decoración y entrada de bolsas, además se estipularon números en la capacidad de las colas, ya que es algo que puede suceder si a la empresa le realizan 
varios pedidos de diferentes clientes y puede generar cuellos de botella, también se realizaron cambios en el tamaño de los lotes y vestidos a cortar.
A continuación se observa la figura 2 del modelo con los cambios implementados

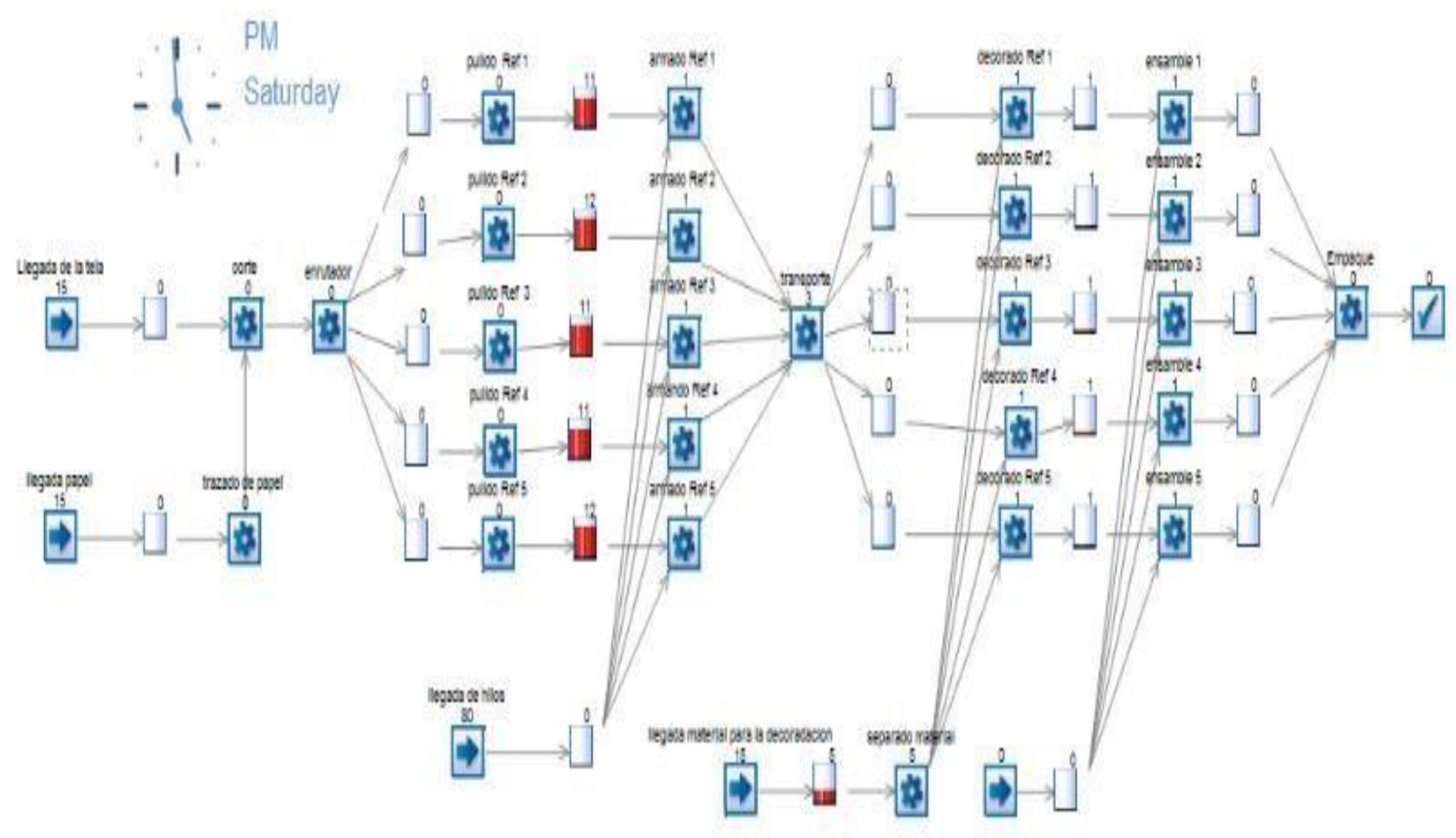

Figura 2: Modelo con cambios del análisis de sensibilidad. Fuente: Elaboración propia

La figura 2 refleja que el modelo es sensible a cambios y que dichos cambios se comportan de una forma similar a la realidad, ya que a una variación en la entrada de materias primas y de la cantidad de unidades que podían estar en cola representan cambios significativos en los resultados obtenidos en la realidad. Además, se observa que la cola del proceso de armado pasa a ser un cuello de botella como lo era antes la del decorado con las modificaciones respectivas.

Tabla 2: Análisis de sensibilidad del modelo Fuente: Elaboración propia

\begin{tabular}{lll}
\hline Parámetro & $\begin{array}{c}\text { Resultado } \\
\text { promedio } \\
\text { obtenido }\end{array}$ & $\begin{array}{c}\text { Resultado } \\
\text { promedio } \\
\text { obtenido (modelo } \\
\text { modificado) }\end{array}$ \\
\hline Entrada de tela & 5 unidades & 15 unidades \\
\hline Tiempo de corte & 6 minutos & 8 minutos \\
\hline $\begin{array}{l}\text { Tiempo de } \\
\text { trazado }\end{array}$ & 1.5 minutos & 4,2 minutos \\
\hline Entrada de hilos & 50 unidades & 150 unidades \\
\hline $\begin{array}{l}\text { Entrada material } \\
\text { decoración }\end{array}$ & 5 unidades & 15 unidades \\
\hline
\end{tabular}

Entrada de bolsas 25 unidades 75 unidades

\begin{tabular}{lcc} 
Cola del corte & 2 minutos & 6 minutos \\
$\begin{array}{l}\text { Cola de la llegada } \\
\text { del hilo }\end{array}$ & 2 minutos & 6,2 minutos \\
$\begin{array}{l}\text { Cola para la } \\
\text { decoración }\end{array}$ & 4 minutos & 12 minutos \\
$\begin{array}{l}\text { Cola de la llegada } \\
\text { de las bolsas }\end{array}$ & 14 minutos & 32 minutos \\
\hline
\end{tabular}

Después de la elaboración detallada del modelo de simulación, su proceso de validación, verificación y análisis de sensibilidad, que es un modelo pertinente y que representa la realidad de la situación problemática de estudio.

\section{RESULTADOS Y DISCUSIÓN}

Se emplea el diseño de experimentos evaluar tres diferentes escenarios propuestos a la empresa y se hace un análisis de los resultados encontrados en cada escenario.

Primer escenario: Contratación una nueva operaria: Dado que el proceso de decoración de vestidos de baño tiene un tiempo de implementación mayor, es necesario considerar la 
idea de contratar una operaria más, a la cual le deben suministrar la capacitación adecuada para la ejecución de las funciones propias de dicho proceso. El objetivo de este escenario es evaluar el sistema con una operaria más en el área de decorado para analizar el comportamiento del mismo.

Segundo escenario: eliminación transporte a Riosucio: Evitar el transporte que se realiza al municipio de Riosucio Caldas y contratar el personal de decoración en el municipio de Itagüí, Antioquia. El objetivo de este escenario es disminuir el tiempo de transporte para agilizar el proceso de decoración.

Tercer escenario: Contratar cinco operarias en decoración: Dado que el proceso de decoración de vestidos de baño tiene un tiempo de implementación mayor y es vital para la culminación de la producción se implementan cinco operarias más, a las cuales se les deben suministrar las capacitaciones necesarias para la ejecución de las funciones para las cuales fueron contratadas. El objetivo es evaluar el sistema con cinco operarias más en el área de decorado para analizar el comportamiento del sistema.

En todos los casos, la longitud de la corrida será un lote de producción y se realizaran 40 corridas. Las variables de estado a analizar son el número de unidades en el sistema y tiempo de proceso de decoración. En la tabla 3, los primeros cinco parámetros corresponden al análisis del primer escenario, los cuatro siguientes corresponden al segundo escenario y los demás corresponden al tercer escenario.

Tabla 3: Comparación de los escenarios descritos. Fuente: Elaboración propia

\begin{tabular}{lll}
\hline Parámetro(actual) & $\begin{array}{c}\text { Valor } \\
\text { numérico } \\
\text { (actual) }\end{array}$ & $\begin{array}{c}\text { Valor numérico } \\
\text { (aplicando } \\
\text { recomendación) }\end{array}$ \\
\hline $\begin{array}{l}\text { Número de } \\
\text { unidades en el } \\
\text { sistema }\end{array}$ & $\begin{array}{l}120 \\
\text { unidades }\end{array}$ & 122 unidades \\
$\begin{array}{l}\text { Tiempo de espera } \\
\text { en la cola de corte }\end{array}$ & 2 minutos & 2 minutos \\
$\begin{array}{l}\text { Número total de } \\
\text { lotes en el sistema }\end{array}$ & $\begin{array}{l}5 \text { lotes }(1 \\
\text { para cada } \\
\text { referencia) }\end{array}$ & $\begin{array}{l}5 \text { lotes (1 para } \\
\text { cada referencia) y } \\
\text { dos unidades más }\end{array}$ \\
$\begin{array}{l}\text { Tiempo de espera } \\
\text { promedio de las } \\
\text { cinco referencias en } \\
\text { proceso de } \\
\text { decorado por }\end{array}$ & 13 minutos & 11 minutos \\
unidad & & \\
$\begin{array}{l}\text { Tiempo de espera } \\
\text { en la cola de } \\
\text { empaque }\end{array}$ & 3 minutos & 2.9 minutos \\
\hline
\end{tabular}

\begin{tabular}{|c|c|c|}
\hline $\begin{array}{l}\text { Número de } \\
\text { unidades en el } \\
\text { sistema }\end{array}$ & $\begin{array}{l}120 \\
\text { unidades }\end{array}$ & 133 unidades \\
\hline $\begin{array}{l}\text { Tiempo de espera } \\
\text { en la cola de corte }\end{array}$ & 2 minutos & 2 minutos \\
\hline $\begin{array}{l}\text { Número total de } \\
\text { lotes en el sistema }\end{array}$ & $\begin{array}{l}5 \text { lotes (1 } \\
\text { para cada } \\
\text { referencia) }\end{array}$ & $\begin{array}{l}5 \text { lotes (1 para } \\
\text { cada referencia) y } \\
13 \text { unidades más }\end{array}$ \\
\hline $\begin{array}{l}\text { Tiempo de espera } \\
\text { de transporte }\end{array}$ & $\begin{array}{l}240 \\
\text { minutos }\end{array}$ & 8 minutos \\
\hline $\begin{array}{l}\text { Número de } \\
\text { unidades en el } \\
\text { sistema }\end{array}$ & $\begin{array}{l}120 \\
\text { unidades }\end{array}$ & 133 unidades \\
\hline $\begin{array}{l}\text { Tiempo de espera } \\
\text { en la cola de corte }\end{array}$ & 2 minutos & 2 minutos \\
\hline $\begin{array}{l}\text { Número total de } \\
\text { lotes en el sistema }\end{array}$ & $\begin{array}{l}5 \text { lotes (1 } \\
\text { para cada } \\
\text { referencia) } \\
\text { y dos } \\
\text { unidades } \\
\text { más }\end{array}$ & $\begin{array}{l}5 \text { lotes( } 1 \text { para } \\
\text { cada referencia) y } \\
13 \text { unidades más }\end{array}$ \\
\hline $\begin{array}{l}\text { Tiempo de } \\
\text { decorado por } \\
\text { prenda promedio de } \\
\text { cinco referencias }\end{array}$ & $\begin{array}{l}302 \\
\text { minutos }\end{array}$ & 50 minutos \\
\hline $\begin{array}{l}\text { Tiempo de espera } \\
\text { promedio de las } \\
\text { cinco referencias en } \\
\text { proceso de } \\
\text { decorado por } \\
\text { unidad }\end{array}$ & 13 minutos & 2.3 minutos \\
\hline
\end{tabular}

Se puede evidenciar en la tabla 3 que el modelo actual presenta tiempos de espera significativos lo cual hace que el sistema se retrase, y al contratar una nueva operaria en confecciones se reduce los tiempos y se aumenta la producción en dos unidades más, sin embargo la mejora es minina, si se relaciona con la inversión necesaria para lograr este incremento en productividad.

En cuanto al transporte a otra ciudad (Riosucio), para realizar el proceso de decoración, al realizar una simplificación del modelo donde se elimine este transporte y se contrate el personal de decorado en el municipio de Itagüí, Antioquia, se puede obtener un ahorro significativo en cuestión de tiempo y de esta manera mejorar la producción.

En el caso de la contratación de cinco operarias se reducen los tiempos de espera y se aumenta la producción en 13 unidades más; sin embargo no hay una mejora significativa en términos de producción, dada la inversión realizada.

Teniendo en cuenta que los costos de fabricación de los vestidos de baño son tan variables debido al incremento del dólar, los únicos costos que se tienen son la mano de obra del área de decorado por lo cual se sugiere realizar capacitaciones a las operarias del área de decorado para que al momento de incluirlas en el proceso ya cuenten con la destreza necesaria para cumplir sus 
funciones. También es necesario realizar una mejora en la distribución del área de trabajo, ya que ergonómicamente no hay una distribución acorde a las necesidades de espacio para la realización de los procesos de ensamble y decorado.

Finalmente, debido a que en el área de decorado se presenta el mayor represamiento de prendas es necesario implementar dos operarias más, para el buen funcionamiento de la planta. La contratación de las operarias es viable financieramente ya que la mano de obra en el municipio de Riosucio, Caldas es muy económica.

\section{CONCLUSIONES}

La simulación del proceso productivo de confecciones, permitió asemejar la estructura y comportamiento del sistema real, con lo cual se logró una mejor comprensión del proceso, de su capacidad y de los recursos u operarios necesarios para llevar a cabo de manera oportuna los pedidos requeridos por sus clientes y por ende incrementar la productividad y rentabilidad de la compañía.

Gracias a los modelos se puede mejorar los procesos de una organización y de esta manera podría encontrarse la mejor forma de atender y realizar un proceso o pedido, el cual va estrechamente ligado con una buena toma de decisiones a nivel organizacional.

Uno de los aspectos más importantes dentro de la simulación es la recopilación y análisis de datos, debido a que son éstos quienes permiten tener un acercamiento al sistema real, realizar pruebas de bondad de ajuste para poder implementar el modelo que se quiere representar y finalmente garantizar una alta confiabilidad de los resultados obtenidos en dicho modelo. Es por ello que la toma de datos es una de los aspectos más críticos pero que más se pasan por alto (no se le presta la debida atención) a la hora de simular.

La alternativa escogida para su implementación dentro de la organización Confecciones es contratar dos operarias más, para el buen funcionamiento de la planta. La contratación de las operarias es viable financieramente ya que la mano de obra en el municipio de Riosucio, Caldas es muy económica y se considera viable contratar dos operarias más aunque elevando un poco costos del producto terminado.

\section{REFERENCIAS}

[1]. Arequipa, M., 2013. Modelo de simulación del sistema de producción para mejorar la gestión de pedidos de la empresa Imagen Textil. Escuela Politécnica Nacional.

[2]. Avila, H.M. \& Vásquez, M.G., 2008. Aplicación de software de simulación como herramienta en el rediseño de plantas de producción en empresas del sector de alimentos. Prospectiva, 6(2), pp.39-45.

[3]. Banks, J., 1998. Handbook of Simulation: Principles, Methodology, Advances, Applications, and Practice 2nd ed., New York: John Wiley \& Sons.

[4]. Bocanegra, C., 2013. Modelo de simulación del abastecimiento de biomasa de caña de azúcar como sistema de soporte a las decisiones de cosecha. Universidad del Valle.

[5]. Gilbert, G.G.N. \& Troitzsch, K.K.G., 2005. Simulation for the social scientist 1st ed., New york: Open University Press.

[6]. Michalus, J.C., Ayala, N. \& Santelices, I., 2007. Elaboración De Té Seco Modeling Applied To the Process of Dry Tea Elaboration. Revista Ingeniería Industrial, 6(1), pp.69-76.

[7]. Morales, Y.L. et al., 2010. Modelamiento de los procesos de producción de bioetanol de primera y segunda generación a partir de caña de azúcar. Etapas; preparación, molienda y clarificación. Umbral Científico, Bogotá Colombia, No.16 p.47(16), pp.47-52.

[8]. Quiroga, O. et al., 2009. Modelos de simulación para el estudio de empresas productivas. Revista Iberoamericana de Ingeniería Industrial, 1(2), pp.02-23.

[9]. Zapata Gaviria, J. \& Peña, G.E., 2006. Simulación de un Proceso de Producción de Marquillas. Avances en sistemas e informatica., 3(1), pp.45-50. 